\title{
SPECTROHELIOGRAPHS FOR THE ULTRAVIOLET
}

\author{
Y. ÖHMAN \\ Stockholm Observatory, Saltsjöbaden, Sweden
}

A brief survey is presented and ground based observations are reported aiming at the design of simple spectroheliographs for space research, particularly in the range 1.500-3.000 ̊.

Two different presumptions have been considered: (a) that a telescope with good pointing control is available, and (b), that the pointing control is only partial or less accurate. One instrument has been constructed by us on each presumption.

1. An Ebert-Fastie spectroheliograph. An Ebert-Fastie system has been built by the writer in collaboration with G. Knutsson \& Co. in Stockholm, using a spherical mirror with a focal length of $1.530 \mathrm{~mm}$ and a diameter of $150 \mathrm{~mm}$. The grating, from Bausch \& Lomb, has a diameter of $80 \mathrm{~mm}$ and 1.800 grooves per $\mathrm{mm}$. In order to reduce the weight of the moving parts as much as possible, or avoid moving parts completely, two different solutions have been tried:

A. A double monochromator with narrow slit using an objective grating with only 300 grooves per $\mathrm{mm}$ and with the dispersion in the same direction as that of the spectrograph (Öhman, 1967; Stenflo, 1968). As can be inferred from Figure 1, a very elongated solar image results, when the two dispersions differ considerably. But even so the solar limb and the spots (visible as elongated objects) can be used for identifying the chords in the solar image corresponding to the different spectrum lines. Compared with the conventional circular image which results when two equal disper-

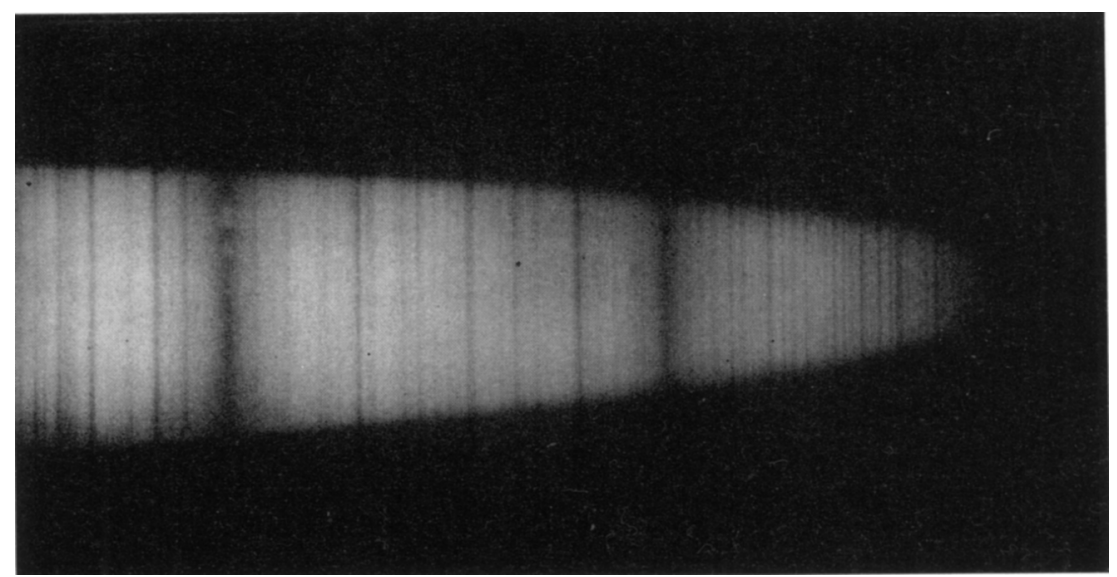

Fig. 1. Elongated spectrum image of the sun obtained on June 24, 1970, in the $\mathbf{H}$ and $\mathbf{K}$ region with an Ebert-Fastie spectroheliograph combined with predisperser. Image size $8 \mathrm{~mm}$. 
sions are used and preferably in a subtractive arrangement, the elongated image has in this case the advantage of covering a greater part of the spectrum. By taking a number of spectra, and displacing the slit or solar image after each exposure, a selected line can be made to correspond to different chords of the solar image, thus giving a scanning by steps.

B. A spectroheliograph with rotating slits. If the entry slit and the exit slit are radially mounted on a metallic disk and so that they form symmetrically situated parts of one and the same diameter, the centre of which 'coincides' with the centre of the grating, the angle of incidence will be practically the same as the angle of diffraction. When giving the metallic disk a slow rotation, and scanning a small solar image, a selected spectrum line will remain in the exit slit producing in this way a conventional spectroheliogram. But if the solar image and its spectrum appear in somewhat different distances from the centre of rotation, a slight drift of the passband will result. Figure 2 shows a spectroheliogram in the $\mathrm{H}$-line of this type (described before by $\mathrm{H}$. Zirin). In spite of the small size of the solar image $(8 \mathrm{~mm})$, Ca II plages are well seen in Figure 2. The principle with rotating slits can be used in spectrohelioscopes as well.

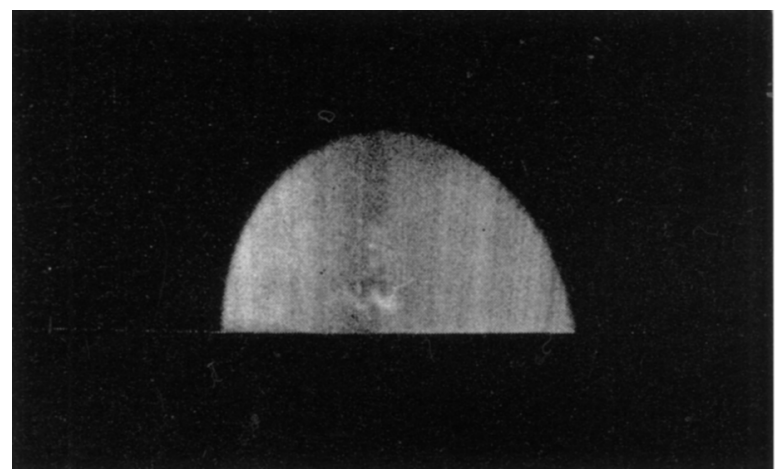

Fig. 2. Spectroheliogram in the Ca II H-line obtained on July 6, 1970, with the Ebert-Fastie instrument showing plages in the centre of the $\mathrm{H}$-line. The predisperser has produced a slight deformation of the image.

2. A spectroheliograph for space vehicles with only partial pointing control using the spin of the vehicle or rotating mirrors.

Since several years the writer has made laboratory experiments on this principle. A prototype has now been built in collaboration with $\mathrm{R}$. Sundqvist in Stockholm. The solar image or its spectrum is formed on a broad slit by means of a $f / 2$ Schmidt mirror (without correcting plate) having a focal length of $200 \mathrm{~mm}$. On the other side of the slit a photographic film will move with more or less the same speed as the image itself.

Figure 3 shows a photograph of this prototype. When equipped with an objective grating the instrument is intended for spectrographic studies in the ultraviolet of solar 
flares. With the small image and fairly good dispersion ( 300 grooves per $\mathrm{mm}$ ) solar flares are expected to appear as brilliant points in a great number of emission lines. The superimposed spectrum of the disk will probably be rather faint. If the motion of the film differs somewhat from that of the image, solar flares will produce broadened emission line spectra, but still of good definition.

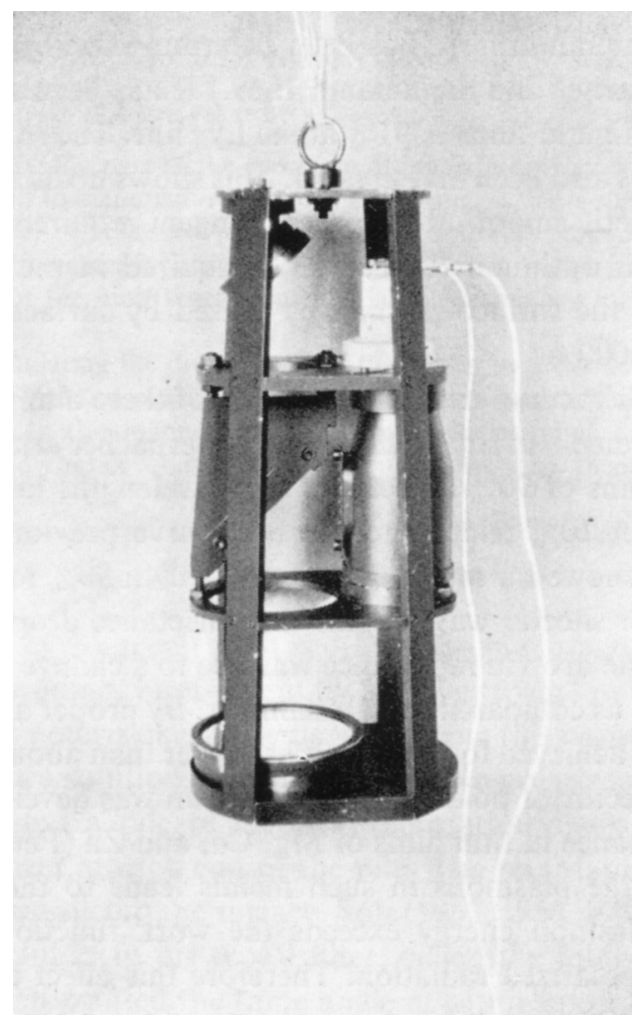

Fig. 3. First prototype of spectroheliograph to be used in a vehicle with only partial pointing control.

\section{References}

Öhman, Y.: 1967, Stockholm Obs. Medd. 175.

Stenflo, J. O.: 1968, Solar Phys. 3, 482. 